\title{
ADDITION OF SODIUM BICARBONATE TO IRRIGATION SOLUTION MAY ASSIST IN DISSOLUTION OF URIC ACID FRAGMENTS DURING URETEROSCOPY
}

\author{
Jessica E. Paonessa ${ }^{1}$, James C. Williams Jr. ${ }^{2}$, James E. Lingeman ${ }^{3}$ \\ ${ }^{1}$ SUNY Upstate Medical University, Dept. of Urology, Syracuse, NY \\ ${ }^{2}$ Indiana University School of Medicine, Dept. of Anatomy and Cell Biology, Indianapolis, IN \\ ${ }^{3}$ Indiana University School of Medicine, Dept. of Urology, Indianapolis, IN
}

\author{
Corresponding Author \\ James E. Lingeman, M.D., FACS \\ Indiana University School of Medicine, Dept. of Urology \\ 1801 North Senate Blvd., Suite 220 \\ Indianapolis, IN 46202 \\ Phone: $317-962-2485$ \\ FAX: 317-962-2893 \\ Email: jlingeman@iuhealth.org
}

Running Head: Dissolving uric acid stones

Key Words: ureteroscopy, uric acid, stones, dissolution, sodium bicarbonate 


\begin{abstract}
Introduction: We hypothesized that adding sodium bicarbonate (bicarb) to normal saline (NS) irrigation during ureteroscopy (URS) in patients with uric acid (UA) nephrolithiasis may assist in dissolving small stone fragments produced during laser lithotripsy. In vitro testing was performed to determine if dissolution of UA fragments could be accomplished within one hour.
\end{abstract}

Materials and Methods: $100 \%$ UA renal calculi were fragmented, filtered, and separated by size. Fragment sizes were $<0.5 \mathrm{~mm}$ and $0.5-1 \mathrm{~mm}$. Similar amounts of stone material were agitated in solution at room temperature. Four solutions were tested (NS, NS+1 ampule bicarb/liter, NS+2, NS+3). Both groups were filtered to remove solutions after fixed periods. Filtered specimens were dried and weighed. Fragment dissolution rates were calculated as percent removed per hour. Additional testing was performed to determine if increasing the temperature of solution affected dissolution rates.

Results: For fragments $<0.5 \mathrm{~mm}$, adding 2 or 3 bicarb ampules/liter NS produced a dissolution rate averaging $91 \pm 29 \%$ per hour. This rate averaged $226 \%$ faster than NS alone. With fragments $0.5-1 \mathrm{~mm}$, addition of 2 or 3 bicarb ampules/liter NS yielded a dissolution rate averaging $22 \pm 7 \%$ per hour which was nearly 5 times higher than NS alone. There was a trend for an increase in mean dissolution rate with higher temperature but this increase was not significant $(P=0.30)$.

Conclusions: The addition of bicarbonate to normal saline more than doubles the dissolution rate of UA stone fragments and fragments less than $0.5 \mathrm{~mm}$ can be completely dissolved within one hour. Addition of bicarb to NS irrigation is a simple and inexpensive approach that may assist in the dissolution of UA fragments produced during ureteroscopic laser lithotripsy. Further studies are needed to determine whether a clinical benefit exists.

\title{
Introduction
}


Approximately $8-10 \%$ of all renal calculi in the United States are composed of uric acid (UA). ${ }^{[1]}$ Numerous studies have shown that the presence of metabolic syndrome (MetS) is associated with an increased risk of developing UA nephrolithiasis. In a retrospective study by Pak et al, $34 \%$ of calculi in diabetic stone formers were composed of UA. In contrast, only $6 \%$ of calculi were UA among nondiabetic stone formers. ${ }^{[2]}$ Metabolic syndrome describes a constellation of disorders which increase an individual's risk of developing diabetes mellitus, coronary artery disease, and stroke. Diagnosis requires the presence of at least 3 of 5 risk factors including central or abdominal obesity, elevated fasting triglycerides, decreased HDL cholesterol, hypertension, or increased fasting glucose. ${ }^{[3]}$ It is estimated that MetS affects nearly $35 \%$ of American adults.

Uric acid stones are relatively fragile ${ }^{[4]}$ and typically produce numerous small fragments when they are broken during endoscopic removal. Small fragments are most likely passed without issue following a procedure but residual fragments may lead to more rapid recurrence of symptomatic stones. ${ }^{[5,6]}$ The incidence of recurrence based on residual stone fragments has not been studied specifically in uric acid stones but it seems logical that any fragments remaining after an endoscopic procedure may lead to a decreased time to recurrence. It can be reasonably argued that residual UA stone fragments could be managed with urinary alkalinization. However, some patients (particularly those with multiple comorbidities) may not be able to tolerate, or may not be compliant with medical management strategies. In this population, it is preferable to achieve a stone-free status.

Ureteroscopy (URS) is generally preferred for the surgical management of renal calculi in obese patients due to body habitus and associated comorbidities. With URS, the UA stones must almost always be broken into pieces for retrieval, typically using laser lithotripsy. This leads to the production of many stone fragments that are too small to be removed and some of these fragments could become nidi for stone regrowth. ${ }^{[5,6]}$ More importantly, basket extraction of numerous fragments is a tedious process, often leading to lengthy procedures in high risk patients. The primary goal using an alkaline irrigant would be to potentially shorten operative times during URS, thus reducing the patients' time under anesthesia. 
We hypothesized that the addition of sodium bicarbonate (bicarb) to normal saline (NS) irrigation during URS in patients with UA nephrolithiasis may assist in dissolving small stone fragments produced during laser lithotripsy by providing an alkaline buffer to accelerate fragment dissolution. In vitro testing was performed to determine if dissolution of UA fragments could be accomplished in one hour or less as this would be an appropriate time to complete an average ureteroscopic procedure.

\section{Materials and Methods}

At our institution, stones removed from patients are routinely analyzed using infrared spectrophotometry to determine mineral composition. After analysis, these specimens are stored in a stone library, de-identified, for research purposes. Renal calculi composed of $100 \%$ UA were selected and mechanically ground into fragments. The fragments were sifted first through a screen with a $1 \mathrm{~mm}$ pore size. Those fragments were then sifted through a second screen with a $0.5 \mathrm{~mm}$ pore size. The fragment sizes used for testing were less than $0.5 \mathrm{~mm}$ and $0.5-1 \mathrm{~mm}$.

Four solutions were prepared for testing. Solution 1 was $0.9 \%$ NS. Solution 2 was $0.9 \%$ NS +1 ampule of bicarb (8.4\% sodium bicarbonate solution; $1 \mathrm{mEq}$ per $\mathrm{ml} ; 50 \mathrm{ml}$ per ampule) per liter (L). Solution 3 was NS +2 ampules of bicarb per liter. Solution 4 was NS +3 ampules of bicarb per liter. All solutions were maintained at room temperature. The $\mathrm{pH}$ of each solution was determined prior to any testing (Table 1).

Similar amounts of stone material $(12.1-18.3 \mathrm{mg})$ were agitated with a magnetic stir bar at 300 revolutions per minute (RPM) in $200 \mathrm{ml}$ of each of the four solutions. Although only $100 \%$ UA calculi were selected for this study, there remains a possibility for stones to have some non-UA components. In order to avoid this as a potential confounder, large stones were chosen allowing for each data set to use fragments from the same specimen for each of the solutions. Fragments less than $0.5 \mathrm{~mm}$ were allowed to dissolve for periods of either 30 or 45 minutes (min). A total of 7 data sets were obtained for fragments less than $0.5 \mathrm{~mm}$ (4 data sets agitated for $30 \mathrm{~min}$; 3 data sets agitated for $45 \mathrm{~min}$ ). Fragments $0.5-1 \mathrm{~mm}$ were allowed to dissolve for 60 min periods. A total of 7 data sets were obtained for fragments $0.5-1$ 
$\mathrm{mm}$. One data set was limited by the size of the original stone, therefore, dissolution of larger fragments $(0.5-1 \mathrm{~mm})$ was only performed in 2 solutions (NS and NS+2). After dissolution, fragments were filtered using acid free filter paper to remove solutions. The filtered specimens were dried in room air for 24 hours and then weighed.

The data were analyzed using analysis of variance (ANOVA) with post-hoc tests accomplished using the Tukey-Kramer HSD (JMP 10, SAS, Cary, NC). Fragment dissolution rates were calculated as percent removed per hour.

Additional testing was performed to determine if raising the temperature of solution would have an effect on the rate of UA dissolution. Fragments less than $0.5 \mathrm{~mm}(0.0247-0.0255$ grams $)$ were agitated at $300 \mathrm{RPM}$ in $200 \mathrm{ml}$ of each of the four solutions. The fragments were allowed to dissolve for 30 min periods. Specimens were then filtered, dried, and weighed as previously described. This experiment was conducted using solutions at room temperature, $37^{\circ} \mathrm{C}$ and $40^{\circ} \mathrm{C}$. Multiple ANOVA (general linear model) was carried out to examine the effect of bicarb content on the solution, controlled for stone source and temperature.

\section{Results}

Addition of bicarb ampules to NS significantly increased the rate of dissolution of uric acid fragments (Figure 1). Three-way ANOVA showed no effect of the different stone sources used for fragments $(P=0.24)$ but highly significant effects of adding bicarb to the solution $(P<0.0001)$ and of fragment size $(P<0.0001)$. With the smallest fragments, the addition of 2 or 3 bicarb ampules to $1 \mathrm{~L} \mathrm{NS}$ resulted in a fragment dissolution rate averaging $91 \% \pm 29 \%$ per hour indicating that such a solution would effectively dissolve all particles of a UA stone smaller than $0.5 \mathrm{~mm}$ within the span of an hour. This dissolution rate was on average $226 \%$ faster than using NS alone. With larger UA fragments (0.5 $1.0 \mathrm{~mm})$, the addition of 2 or 3 bicarb ampules to $1 \mathrm{~L} \mathrm{NS}$ yielded a lower rate of dissolution $(22 \% \pm 7 \%$ per hour) than with the smaller fragments but the rate was still almost 5 times higher than that measured in larger fragments with NS alone. 
In the experiment to test whether raising the temperature of the solution would further increase the rate of dissolution of UA fragments, only the smaller size range was used. Three-way ANOVA again showed no effect of the different stone sources used for fragments $(P=0.10)$, as well as a significant effect of the addition of bicarb to the NS $(P<0.0001)$. There was a trend for an increase in mean rate with higher temperature but this increase was not significant $(P=0.30)$. Overall, the mean rates of dissolution (all temperatures together) were remarkably close to the data in Figure 1 for smaller fragments: $45 \pm 4$, $85 \pm 10,100 \pm 17$ and $103 \pm 13 \%$ per hour for the NS, NS $+1, N S+2$, and NS+3 solutions, respectively.

\section{Discussion}

Three urinary abnormalities are observed in patients with UA calculi. ${ }^{[1,7]}$ The most important finding is that of a low urinary $\mathrm{pH}$ (usually less than 5.5).$^{[1,7,8]}$ MetS is associated with significantly lower urine $\mathrm{pH} \cdot{ }^{[1,7,8]}$ A second factor is low urine volume (less than $2 \mathrm{~L}$ per day).$^{[1]}$ Hyperuricosuria is a third abnormality that is most commonly the result of excessive consumption of dietary purines (usually animal protein). It should be noted that the majority of UA stone formers have normal UA excretion. ${ }^{[1,7]}$ Low urinary $\mathrm{pH}$ in individuals with MetS is due to insulin resistance which causes an increased net acid excretion and impaired buffering (due to defective renal ammoniagenesis)..$^{[1,7,8]}$ The pK of UA in urine is $5.35^{[7]}$ which explains the resultant precipitation of UA crystals at a low urinary $\mathrm{pH}$.

Surgical management of UA nephrolithiasis in the obese population can be particularly challenging due to body habitus and associated comorbidities. ${ }^{[9-14]}$ Extracorporeal shock wave lithotripsy is often precluded by weight limitations of the lithotriptor and a large skin-to-stone distance. ${ }^{[9-14]}$ Most lithotriptors have a maximum focal point of 12 to $14 \mathrm{~cm} .{ }^{[10]}$ Percutaneous nephrolithotomy (PCNL) usually requires a patient to be positioned prone and such positioning can lead to respiratory compromise and impaired venous return. ${ }^{[9]}$ Patient size/habitus may exceed the comfort level of the urologist and may also require longer instruments. ${ }^{[10]}$

In 2012, Aboumarzouk and Monga et al published a meta-analysis concerning the role and safety of URS for stone management in obese patients. A total of 7 articles were included for review. ${ }^{[9]}$ Mean 
body mass index (BMI) was 42.2 (range 30.13 - 65.2). All patients were treated with flexible URS and mean operative time was $97.1 \mathrm{~min}$ (range 30 - 275). Overall success rate (defined as stone free or less than $2 \mathrm{~mm}$ ) was $87.5 \%{ }^{[9]}$ A sub-group analysis found higher success rates when treating ureteral stones and stones less than $2 \mathrm{~cm}$. Two of the reviewed articles reported 2.6 and 2.3 procedures per patient. ${ }^{[9]}$ The overall complication rate was $11.4 \%$. However, most required only antibiotics or analgesics (Clavien Grade 2). The only grade 3 complication was a ureteral perforation and no grade 4 or 5 complications were reported. ${ }^{[9]}$ A recent multicenter comparison study by Chew et al compared the safety and effectiveness of ureteroscopic laser lithotripsy in obese and normal weight patients. The authors found obese patients had equivalent stone free rates compared to normal weight patients following URS. ${ }^{[10]}$ Increased BMI was associated with a prolonged hospital stay but an equivalent complication rate to nonobese patients. ${ }^{[10]}$

URS can be considered a first line therapy in the surgical management of renal calculi in obese patients. ${ }^{[11-13]}$ Treating obese individuals is often problematic for the urologist due to their greater risk of anesthetic complications. ${ }^{[9,12-14]}$ Therefore, it is desirable to develop techniques that could expedite the removal of stone material during URS which may allow for decreased time under anesthesia and minimize the risk of associated morbidity. A reasonable next step would be to determine whether altering the $\mathrm{pH}$ of irrigation fluid can reduce operative times during ureteroscopic management of large UA stones. The data in the present study suggests that addition of bicarb to the saline irrigation used for URS could increase the UA stone fragment dissolution rate twofold to fivefold over what would occur using NS alone. Moreover, it appears from the dissolution rates measured that all of the fragments smaller than $0.5 \mathrm{~mm}$ could be dissolved during an hour-long case. If our hypothesis is found to be correct, it could potentially expand the indications for URS, consequently reducing the need for more invasive procedures like PCNL.

This in vitro study does have limitations. In particular, the number of stone samples tested was small and, thus, may not be representative of all UA stones. On the positive side, no effect of the different stone sources used in this study was seen. In this study, a static model was used to evaluate UA 
fragment dissolution. This model could not account for the effects of continuous irrigant flow or the relatively acidic urine production that would occur during an actual ureteroscopic procedure. Stirring fragments in a fixed volume probably underestimates the dissolution rates that would be seen with continuous flow of irrigant. Ultimately, this method needs to be tested in a clinical setting which could also allow the asking of some additional questions: Will higher stone free rates be achieved in UA stone patients treated with URS when bicarb is added to the saline irrigant? Can such interventions during URS produce longer intervals between stone events or increased time for stone regrowth?

The primary goal of this study is to determine whether increasing the $\mathrm{pH}$ of irrigation fluid can increase the dissolution rate of UA fragments. This concept could be applied clinically to test if alkaline irrigation fluid can reduce operative times during ureteroscopic management of large UA stones.. With a future clinical study in mind, sodium bicarbonate was chosen as a readily available, inexpensive, alkaline buffer to accelerate UA fragment dissolution. In designing a clinical study, the authors recommend the use of large ureteral access sheaths to facilitate the removal of larger stone fragments and decrease operative times. Employing an access sheath allows the intrarenal pressure to remain low, thus reducing the risk of significant fluid absorption. In 2004, Cybulski et al published a study suggesting that routine URS is associated with minimal systemic fluid absorption, even if ureteral perforation occurs. ${ }^{[15]}$ Therefore, complications secondary to hypernatremia should be extremely low. However, the risk is not zero and patient safety is of chief importance. For a clinical study, the authors would utilize NS in the control arm and an appropriate amount of sodium bicarbonate (e.g. 2 ampules) added to a hypotonic irrigation fluid (e.g. 1/2 NS) to approximate normal osmolality in the high $\mathrm{pH}$ arm.

\section{Conclusions}

The addition of bicarbonate to normal saline more than doubles the dissolution rate of UA stone fragments and fragments less than $0.5 \mathrm{~mm}$ can be completely dissolved within one hour. Addition of bicarb to NS irrigation is a simple and inexpensive approach that may assist in the dissolution of UA 
fragments produced during ureteroscopic laser lithotripsy. Further studies are needed to determine whether a clinical benefit exists.

Disclosures: The authors have nothing to disclose. 


\section{References:}

1) Maalouf NM. Metabolic syndrome and the genesis of uric acid stones. J Ren Nutr 2011; 21(1):128-131.

2) Pak CY, Sakhaee K, Moe O, et al. Biochemical profile of stone-forming patients with diabetes mellitus. Urology 2003; 61:523-527.

3) Executive summary of the third report of the National Cholesterol Education Program (NCEP) expert panel on detection, evaluation and treatment of high blood cholesterol in adults (Adult Treatment Panel III). JAMA 2001; 285:2486-2497.

4) Williams JC Jr, Saw KC, Paterson RF, et al. Variability of renal stone fragility in shock wave lithotripsy. Urology 2003; 61:1092-1096.

5) Streem SB, Yost A, Mascha E. Clinical implications of clinically insignificant stone fragments after extracorporeal shock wave lithotripsy. J Urol 1996; 155:1186-1190.

6) Rebuck DA, Macejko A, Bhalani V, et al. The natural history of renal stone fragments following ureteroscopy. Urology 2011; 77:564-568.

7) Asplin JR. Obesity and Urolithiasis. Advances in Chronic Kidney Disease 2009; 16(1):11-20.

8) Sakhaee K, Maalouf NM. Metabolic syndrome and uric acid nephrolithiasis. Seminars in Nephrology 2008; 28(2):174-180.

9) Aboumarzouk OM, Somani B, Monga M. Safety and efficacy of ureteroscopic lithotripsy for stone disease in obese patients: a systematic review of the literature. BJUI 2012; 110:E374-E380.

10) Chew BH, Zavaglia B, Paterson RF, et al. A multicenter comparison of the safety and effectiveness of ureteroscopic laser lithotripsy in obese and normal weight patients. J Endourol $2013 ; 27(6): 710-714$.

11) Bultitude MF, Tiptaft RC, Dasgupta $P$, et al. Treatment of urolithiasis in the morbidly obese. Obes Surg 2004; 14:300-304.

12) Dash A, Schuster TG, Hollenbeck BK, et al. Ureteroscopic treatment of renal calculi in morbidly obese patients: a stone-matched comparison. Urology 2002; 60:393-397.

13) Andreoni C, Afane J, Olweny E, et al. Flexible ureteroscopic lithotripsy: first-line therapy for proximal ureteral and renal calculi in the morbidly obese and superobese patient. $J$ Endourol 2001; 15:493-498.

14) Nguyen TA, Belis JA. Endoscopic management of urolithiasis in the morbidly obese patient. $J$ Endourol 1998; 12:33-35.

15) Cybulski P, Honey RJ, Pace K. Fluid absorption during ureterorenoscopy. J Endourol 2004; 18(8):739-42. 